\title{
La participación de las mujeres en el desarrollo rural y el bienestar social
}

Marta Gil Lacruz, Alicia Izquierdo \& Pilar Martín

La mujer en el entorno rural se encuentra en una situación de vulnerabilidad que obliga a los agentes sociales a la articulación de medidas específicas de desarrollo y participación. Al hecho de encontrarse en un enclave económicamente desprotegido se suele sumar una cultura compartida reticente al cambio y a la innovación en los roles de género. Pero el bienestar social y el desarrollo comunitario no son posibles en nuestro entorno globalizado sin contar con el bienestar individual y la importancia de su capital humano y social. El artículo reflexiona sobre estos temas y aporta posibles estrategias de intervención.

desarrollo rural / género / participación comunitaria

Women's involvement in rural development and the social welfare

Women are frequently situated at a risky situation at the rural social environment. Social agents aware of this situation, have to design specific programs to them in order to improve her development and participation. They have to affront not only the economical decadency of their communities but the native culture resistance to social innovation and traditional gender roles change. But in our globalize world, welfare is not possible without taking into account personal wellbeing and human and social capital importance. This article deeps into these topics and suggests possible intervention strategies.

rural development / gender / community participation 


\section{INTRODUCCIÓN}

Según Izquierdo (2006), numerosos estudios atribuyen a la mujer el nacimiento de la agricultura. Seguramente, fue una mujer la que tuvo la paciencia y la perspicacia suficiente como para sentarse a observar el crecimiento de las plantas a partir de las semillas. Y sembrar.

Hace muchos miles de años fue la mujer quien impulsó el sedentarismo, un nuevo modelo de producción, y el asentamiento de nuestros pueblos. La innovación de esta técnica contribuyó -junto con otros muchos factores- a nuestro crecimiento demográfico, mejoró nuestra esperanza de vida, cambió nuestros estilos de vida y alimentación, generó un tiempo y un espacio nuevos, facilitó el ocio y la creatividad en el arte y la ciencia, etcétera.

Hoy como ayer, nos preguntamos cuál es el papel de la mujer en el mantenimiento y la mejora de un medio rural dinámico y próspero. La pregunta es personal pero también institucional, ya que diversos organismos internacionales, como la Organización de las Naciones Unidas, se hacen eco del protagonismo femenino en todos sus programas de desarrollo, por ejemplo: microcréditos, erradicación del hambre, mejora de la salubridad del entorno, capacitación comunitaria, etcétera.

\section{LA SITUACIÓN DE LA MUJER EN EL ENTORNO RURAL}

Aún hoy la visibilidad social de los hombres sigue siendo superior a la de las mujeres. Si hablamos de un entorno rural desprotegido y despoblado, el anonimato de este colectivo está más que garantizado.

Por ejemplo, en una provincia rural española como Teruel, y en su terreno laboral, encontramos dos datos aparentemente contradictorios. Por una parte, la tasa de desempleo es menor que las tasas aragonesas y españolas; por otra, la tasa de actividad de sus mujeres y de sus jóvenes es considerablemente inferior a la del promedio español (García, 2004).

La carencia de oferta de trabajos especializados, la reducida contribución al PIB sectorial de la población agraria activa, los elevados porcentajes de desempleados, etcétera, condicionan aún más la migración de los habitantes autóctonos de la provincia mencionada (Frutos y Rubio, 2000). El sector turístico y los proyectos de pequeña envergadura (empresas artesanales y pymes) han consumido gran parte de la inversión europea.

Pero este fenómeno tampoco es nuevo. La masiva emigración del campo a la ciudad, que se desarrolló durante gran parte del siglo pasado, no es sino consecuencia lógica de estas dificulta- 
des. El abandono que un gran número de mujeres hicieron del ámbito rural supuso una seria amenaza para muchos pueblos, los cuales se encontraron en una situación terminal por no poder garantizar el relevo generacional.

Este éxodo se caracterizó no solo por la desagrarización del campo, sino también por la búsqueda por parte de la mujer de nuevas formas sociales de relación, quizás más justas, igualitarias y previsibles en un entorno urbano, más complejo, culto y cosmopolita.

Y esto es así porque además de un escenario adverso en cuanto a realización profesional, las mujeres -en el entorno rural- han tenido que hacer frente a la presión de unas creencias tradicionales y estereotipadas sobre lo que es "femenino" y "masculino", que solo recientemente se han empezado a cuestionar. La defensa de valores y cualidades supuestamente "femeninas", como la pasividad, la paciencia, la abnegación, la ternura, la orientación hacia la familia y la emotividad, han contribuido a confinar aún más a las mujeres rurales en su entorno doméstico y en una esfera privada en la que ejercen de cuidadoras o de mesoneras.

El ideal de madre y esposa ha afectado de manera discriminatoria a una parte importante de la ciudadanía, que ve en el ámbito familiar el lugar de su realización personal. Lo romántico y el maternaje no condicionan del mismo modo a sus compañeros, ni con la misma intensidad. Y estas diferencias se agudizan aún más en enclaves que se caracterizan por la vigencia y la defensa de los valores tradicionales.

No se trata simplemente de reivindicar el hecho de que las mujeres juegan un papel fundamental como mediadoras en relación con los ámbitos educativos, sanitarios y de los servicios sociales, sino que estamos hablando de verdaderas ciudadanas con una serie de derechos y deberes adquiridos y propios.

Este reconocimiento tampoco puede olvidar el papel de todas aquellas mujeres que provienen de otros países, etnias o religiones y que comparten en nuestro mundo rural su cultura. También ellas son artífices del mantenimiento de nuestra economía y juegan un papel estratégico en el desarrollo de nuestra región.

Por eso resulta urgente el cambio de los modelos de desarrollo rural y de bienestar social diseñados exclusivamente por los varones. En muchos de ellos no se han contemplado las posibles aportaciones de sus compañeras ni se ha valorado el impacto de la implementación de sus programas en las relaciones de género. Muchos de ellos se han centrado en el crecimiento economicista, obviando los aspectos socioculturales del desarro1lo, como las creencias, los valores y las normas reguladoras de los sistemas sociales. 
DEFINICIÓN DEL BIENESTAR INDIVIDUAL Y SOCIAL: EL DESARROLLO DEL CAPITAL SOCIAL

En la necesidad de valorar este predominio masculino también es prioritario revisar nuestra definición de bienestar. Su evaluación a menudo se encuentra en la discrepancia o congruencia entre lo que uno tiene o desea, la comparación con los demás, lo que esperaba y lo que obtuvo, lo que necesita y lo que tiene (Michalos, 1995). Este tipo de ecuaciones nos remite primero a las posibilidades actuales del individuo y, posteriormente, a las capacidades con las que ciertas condiciones vitales -por ejemplo en el entorno rural- nos facilitan o impiden un nivel determinado de calidad de vida (Blanco, Rojas \& Corte, 2000).

Incluso si adoptamos un enfoque individualista de la satisfacción de las necesidades vitales como fuente de felicidad, en la evaluación personal del bienestar entrarían factores sociales como las aspiraciones, las comparaciones con otras personas o los valores sociales del camino que hay que seguir para alcanzarla (Díaz \& Blanco, 2005). Y esto es así porque vivimos inmersos en un mundo de sistemas sociales: familia, amigos, compañeros de trabajo, vecinos. El mundo rural no es ajeno a los procesos de internacionalización. La informática y los mass media comunican, como en una verdadera correa de transmisión, los cambios sociales de lugares remotos. El mundo exterior está cada vez más presente en el ámbito campesino e incide sobremanera sobre sus propios procesos y dinámicas.

Nuestro bienestar individual depende, por tanto, de lo que nos ocurre directamente (y cómo lo interpretamos) y del intercambio que mantengamos con los que nos son próximos y los que no necesariamente lo son pero influyen en nuestra vida. Nuestro bienestar se ve potenciado cuando la sociedad de adscripción permite su desarrollo activo (Herrero, 2004). De ahí que en el análisis crítico de la riqueza de una comunidad se deba contemplar siempre la combinación de nivel macro (cómo afectan las políticas internacionales, económicas, laborales, etcétera) y micro (importancia de la identidad, de la trayectoria concreta de reinserción laboral).

Una vez garantizadas las condiciones básicas de subsistencia, el desarrollo del bienestar comunal depende no solo del crecimiento de las dimensiones económicas sino también de variables como la innovación en la tecnología disponible y el capital humano y social, en los que interviene de manera privilegiada el factor humano y relacional (Altman, 2001; Martín \& Agut, 2005).

La Organización para la Cooperación y el Desarrollo (OCDE) (2001) define el capital humano como el conocimiento y estado de salud del individuo, mientras que el concepto de capital social incorpora las normas y redes 
que facilitan la cooperación entre los grupos y dentro de estos. Putnam (1993) dimensiona en el capital social de una comunidad las siguientes características:

- La existencia de una red densa de organizaciones locales, comunitarias y asociativas.

- Elevados niveles de compromiso cívico y participación en estas comunidades.

- Una identidad local fuerte y positiva que comparta un elevado sentido de solidaridad y equidad entre sus miembros.

- Normas generalizadas de confianza y apoyo social entre sus miembros, independientemente de su conocimiento mutuo.

El capital social y el estándar de vida de un territorio repercuten en la elección individual de una vivienda, de un puesto de trabajo, del estilo de vida que se mantiene y cómo y con quién nos relacionamos. De igual modo, las creencias culturales, por ejemplo, sobre el lugar de la mujer en la sociedad y su papel en la familia modela los roles y conductas que se adoptan y distribuyen socialmente (cuidadora de otros miembros, veladora de su salud y educación, sacrificada de su propio bienestar).

Lo que ya no es evidente es la forma específica de articular, de un modo coherente, el conjunto de indicadores y demandas potenciales. A pesar de sus enormes posibilidades, su carácter multidimensional y multidisciplinar, el bienestar requiere de un marco de análisis integrador y en el que se complementen al unísono su vertiente individual con su naturaleza social y grupal (Gil Lacruz, 2007).

Una posible definición operativa de bienestar social debe de integrar al menos cinco dimensiones (Blanco \& Díaz, 2005):

- Integración social: definida como la evaluación de la calidad de las relaciones que mantenemos con la sociedad (el sentimiento de pertenencia y de identificación).

- Aceptación social o la valoración que hacemos de las personas y de la naturaleza humana en general. Frente al rechazo machista de una escala de valores y una esfera afectiva que nos complementa y refuerza, y que se considera poco productiva.

- Contribución social, la cual incluye la sensación de que tenemos una función y un sentido de utilidad social. Que en el entorno rural trasciende de las cuatro paredes de una vivienda rústica, o de su nuevo estatus de reserva de la biosfera para urbanitas estresados.

- Actualización social o la confianza desarrollada en el futuro de la sociedad y en sus posibilidades de crecimiento y desarrollo. Este factor se relaciona directamente con la idea 
de progreso y de cambio social y con la evidencia de que el desarrollo económico de la comunidad, la igualdad social, el acceso educativo y el respeto a los derechos y libertades cívicas influyen directamente sobre nuestro bienestar individual.

- Coherencia social, que determina el orden organizativo y el funcionamiento del mundo social. Si las condiciones de trabajo son adecuadas, pero en la esfera del hogar aún se está muy lejos de compartir responsabilidades, la coherencia de nuestras políticas sociales seguirá siendo discutible.

El desarrollo rural, en definitiva, constituye un proceso de crecimiento cuyo objetivo consiste en hacer posible el progreso global de la población rural en su dimensión cultural, educativa, social, económica y política. Este progreso se concreta en el desarrollo de la cultura, la educación, la economía, el empleo, los servicios sociales, la cohesión y el fortalecimiento de la sociedad civil, la mejora democrática, etcétera.

El fin del desarrollo rural y de los diferentes colectivos que lo integran no es exclusivamente la acumulación de la riqueza, los adelantos tecnológicos y la maximización y optimización de los recursos. Este crecimiento es un medio al servicio de la potenciación integral de la persona y su comunidad.

Esta declaración de intenciones las observamos en el redescubrimiento actual del medio rural, asociado cada vez más a nuevos valores de paz, naturaleza y tranquilidad. Se reivindica la pervivencia de unas costumbres auténticas y raíces de nuestra identidad, de un patrimonio cultural y de una herencia histórica, artística y natural, pero también se defiende la disponibilidad de un capital social, de unas redes de apoyo y de una revalorización de lo femenino, que pueden constituir un recurso contra el estrés y el individualismo propio de la deshumanización actual de algunos núcleos urbanos.

Así como la ausencia de la mujer fue clave en la crisis de la ruralidad y en la despoblación de nuestras comarcas, su presencia y participación resulta prioritaria en estos procesos de recuperación a los que asistimos hoy. Y ello por varias razones (Díaz, Gil, Izquierdo, Jiménez, Lucas, Pérez \& Rezusta, 2005):

- No se puede hablar de reparación demográfica sin la participación de la mujer, sobre todo de la mujer joven. Si no se alcanza un equilibrio entre sexos para que las jóvenes puedan quedarse en su pueblo y fundar una familia, el concepto de sustentabilidad de la población rural no tiene ningún sentido.

- Para que la mujer opte por asentarse en el entorno rural resulta fundamental priorizar su participación equitativa en el mundo laboral. La excesiva masculinización agraria y los puestos de trabajo asociados al 
rol masculino hacen que la mujer se sienta con frecuencia excluida del ámbito productivo. Las mujeres con ideas innovadoras y nuevos proyectos productivos tienen dificultades para salir adelante en una cultura que las confina a lo doméstico y a "la pata quebrada". La ausencia de modelos previos también funciona en contra de ellas.

- La cultura de la diferencia y de la segregación, que separa tajantemente los ámbitos domésticos y sociales, privados y públicos, cerrados y abiertos, debe dar paso a un sistema de valores más flexible y proclive a la participación fluida de las mujeres en distintos escenarios, relaciones y sistemas.

\section{La ARTICULACIÓN DE LA PARTICIPACIÓN CIUDADANA}

Según Jiménez y Gil (2007), la participación se convierte en el medio privilegiado por el cual las personas pueden acceder y controlar los recursos de su entorno. Implica tanto la sensibilización social sobre su importancia como el compromiso individual de las personas. Toma de conciencia, participación y cambio constituyen una tríada indisoluble pero de difícil consecución en enclaves rurales caracterizados por la indefensión, la baja autoestima o la desigualdad de oportunidades para su ejercicio.
Podemos articular la participación en los siguientes principios operativos (Ayuntamiento de Zaragoza, 2006):

- Compartir las similitudes. Hay problemas que todos los ciudadanos padecen y deben encaminarse todos los esfuerzos de una manera conjunta, con el fin de mejorar las condiciones de vida para todos. El tejido asociativo, tanto formal como informal, puede constituirse en un vehículo interesante para gestionar las soluciones de un modo creativo y basado en la convivencia. En el terreno rural el asociacionismo de mujeres tiene un carácter cultural y recreativo, pero de ahí surgen también ideas innovadoras de producción y capitalización económica.

- Potenciar, valorar y respetar las disimilitudes, como una manera de hacer ciudadania. Por tanto, hay que reconocer el liderazgo y los mecanismos propios de representación. Hemos analizado que hablar de mujer y de desarrollo rural no nos remite exclusivamente a los parámetros economicistas $\mathrm{o}$ de empleo femenino. Interesan también las posibilidades de desarrollo personal y de autorrealización de las mujeres y de los diferentes colectivos que componen una comunidad, pretendiendo alcanzar un modelo integral de bienestar.

- Implicar a la sociedad civil en las iniciativas, propuestas y soluciones. En muchos casos, esto puede supo- 
ner animar a las personas a encontrar espacios sociales donde puedan hacer llegar sus quejas y sugerencias, tanto a través de organizaciones formales como informales existentes o que se pueden constituir ad hoc, como asociaciones vecinales, comarcales, de consumidores, organizaciones no gubernamentales, sindicatos, asociaciones de padres y madres, etcétera.

- Fomentar la responsabilidad compartida para asegurar una convivencia pacífica. En este sentido, también resultan necesarios los estudios e investigaciones que profundicen en los factores psicosociales que se encuentran presentes en dicha dinámica preventiva, por ejemplo al compartir las tareas en el hogar, el cuidado de enfermos, la educación de los hijos, pero también la reparación de pequeñas averías, la contabilidad de la economía familiar, etcétera.

- Potenciar los canales participativos del asociacionismo, así como gestionar la posibilidad de ser entidades consultivas, formativas, de autoempleo y de mediación, a través del establecimiento de convenios que desarrollen programas sociales, con objetivos, medidas concretas, evaluación y seguimiento conjunto de resultados por parte de los técnicos competentes. No cabe duda de que el nivel de asociacionismo de un territorio puede ser un indicador clave del desarrollo democrático de la sociedad en la que se inserta.

Nos encontramos en un momento trascendental de cambio. El viento corre hacia la corresponsabilidad y la igualdad. La participación de las mujeres se fomenta desde los organismos públicos, como la reciente aprobación en España de la Ley Orgánica para la Igualdad Efectiva de Mujeres y Hombres. Dicha ley ha resultado necesaria, ha supuesto una acción normativa dirigida a combatir todas las manifestaciones aún subsistentes de discriminación directa o indirecta por razón de sexo. Pretende promover la igualdad real entre mujeres y hombres, con remoción de los obstáculos y prejuicios sociales que impiden alcanzarla. Esta exigencia se deriva de nuestro ordenamiento constitucional e integra un genuino derecho de las mujeres a su participación plena en el bienestar individual y social.

En este contexto, la participación de la mujer rural cobra un protagonismo propio. Además de beneficiarse de las diferentes acciones propuestas en la ley de manera transversal, esta le concede un espacio específico. Así, el artículo 30 está dedicado al "desarrollo rural" y en él se recogen diferentes medidas cuyo fin último es el reconocimiento y la potenciación de la mujer rural en su medio. Destacamos los siguientes aspectos (Martínez \& Izquierdo, 2007): 
- Desarrollo de la figura jurídica de la titularidad compartida, para que se reconozcan plenamente los derechos de las mujeres en el sector agrario, la correspondiente protección de la seguridad social, así como el reconocimiento de su trabajo.

- Acciones dirigidas a mejorar el nivel educativo y de formación de las mujeres, especialmente las que favorezcan su incorporación al mercado de trabajo y a los órganos de dirección de empresas y asociaciones.

- Encomienda a las administraciones públicas a promover nuevas actividades laborales que favorezcan el trabajo de las mujeres en el mundo rural.

- Atención al desarrollo de una red de servicios sociales para atender a menores, mayores y dependientes como medida de conciliación de la vida laboral, familiar y personal de hombres y mujeres en el mundo rural.

- Fomento del acceso a las tecnologías de la información y la comunicación mediante el uso de políticas y actividades dirigidas a la mujer rural y a la aplicación de soluciones alternativas tecnológicas allá donde la extensión de estas tecnologías no sea posible.

Se trata, desde luego, de instrumentos muy necesarios que responden a las "debilidades" detectadas para la plena participación social entre las mujeres de este medio. Ahora debemos de ser capaces de promover estrategias, políticas y condiciones que faciliten de manera operativa esta pretendida igualdad del individuo y de los grupos.

\section{PolítICAS Y ESTRATEGIAS DE INTERVENCIÓN}

Partimos de la base de que deben ser las administraciones públicas las que propicien políticas concretas de integración social, por la cercanía a las demandas y necesidades de sus ciudadanos. Las políticas de desarrollo rural deben aspirar a satisfacer dichas demandas, identificando también aquellos condicionantes que impiden el pleno desarrollo de la mujer y propician su emigración forzada o deseada.

Se requiere transversalidad en las acciones y verticalidad en la coordinación entre distintas administraciones para mejorar la calidad de vida de las ciudadanas. Especialmente importante sería el principio de normalización relacionnado con los posibles servicios que se generen. Hay que evitar la creación de circuitos estigmatizantes (tan frecuentes para los colectivos desfavorecidos y desde los servicios sociales o asistenciales).

Así como no podemos hablar de un único colectivo de emigrantes, de personas de tercera edad o de jóvenes, deberíamos evitar caer en la simplificación de un único modelo de políticas de igualdad para un único y ficticio colectivo femenino en lo rural. 
La diversidad, que es sinónimo de complementariedad y enriquecimiento, no siempre es igual a integración. La convivencia en la diversidad no es sencilla, y tampoco suele estar exenta de conflictos, pero por lo menos garantiza que la comunicación será un requisito de obligado cumplimiento.

Dentro de esta diversidad debemos hablar también de desigualdad; las mujeres inmigrantes, extranjeras, maltratadas, en situaciones de ilegalidad, etcétera, también residen en nuestros pueblos y componen nuestro tejido social.

Por el contrario, y añadiendo más complejidad al ya de por sí delicado crisol cultural que se da en nuestros pueblos hoy en día, caer en el polo contrario y sustentar la intervención sobre pilares excesivamente individualistas supondría caer en el particularismo y obviar la existencia de un proyecto de desarrollo colectivo en el entorno rural.

Para Vásquez (2000) el criterio clave de la intervención en nuestros pueblos se encuentra en el desarrollo endógeno, en el que lo social y lo económico se funden. Los principios en los que se fundamenta son dos:

- Concienciación de la importancia que tienen los sistemas de producción local en los procesos de crecimiento económico en los que se integran lo social y lo ecológico. "Pan para hoy y hambre para mañana" no es su lema. La sostenibilidad pre- tende garantizar la cobertura de las necesidades del presente sin comprometer las de las generaciones del futuro.

- Consideración imprescindible de la iniciativa local, como forma preferente de la política de desarrollo, es decir, la capacidad de liderar el propio desarrollo. Es imprescindible que exista un acuerdo tácito o expreso de los actores económicos y sociales (hombres y mujeres) sobre las prioridades y caminos que se deben seguir.

Cohesión y participación forman parte del capital social con el que cuentan los grupos y las asociaciones de mujeres para comprometerse con unos objetivos y conseguir mayores niveles de control sobre sus circunstancias (Campbell, 2000). Esta evidencia se manifiesta a su vez en el concepto de capacitación y potenciación comunitaria.

La potenciación comunitaria presenta dos elementos esenciales para su desarrollo: la autodeterminación o autodirección, es decir la determinación de cada uno sobre su propia vida, y de ahí el sentimiento de control personal, y la determinación social o participación democrática en la vida de la propia comunidad, relacionada con el interés por la influencia social real, el poder político y la defensa de los derechos legales (Musitu y Buelga, 2004). Esta potenciación se logra a través de la participación comunitaria. 
El papel del especialista en igualdad de oportunidades, el de desarrollo rural y el científico de lo social han de coincidir en el posicionamiento de su rol profesional comprometido con la crítica y la denuncia de situaciones de injusticia y de desigualdad, valiéndose de herramientas metodológicas, que, por su rigor y coherencia, pretenden ser científicas (Bourdieu, 2002).

Es necesario plantearse la adecuación de la metodología de intervención a los retos de una realidad demográfica cambiante. Entre sus posibles objetivos (por ejemplo los de la Concejalía de Educación y Acción Social del Ayuntamiento de Zaragoza, 2006) figuran la ampliación de su metodología de intervención a cuestiones relativas a la investigación-acción y alternar los niveles de intervención: individuales, grupales y comunitarios.

- Aunque hablemos de actuaciones individuales no podemos eludir la importancia de la reflexión sobre nuestra red de relaciones sociales. Por ejemplo, el entorno laboral puede constituir un escenario privilegiado para desarrollar nuestro capital humano (mediante programas formativos y educativos adecuados), y social (redes de relaciones sociales, como programas de cooperativismo, facilidades para la creación de empresas). Pero en el ámbito rural, la mujer debería encontrar no solo un sitio donde trabajar, sino su sitio de desarrollo y realización. Por ello, ningún programa dirigido "hacia" o "para" la mujer podrá ser eficaz si no cuenta con la participación de sus protagonistas.

- Entre las actuaciones grupales destacamos la importancia de la adquisición de competencias y habilidades que favorezcan la integración, a través de la información y asimilación de recursos, derechos y obligaciones, la comunicación con otras personas que también han llevado a cabo procesos personales de resolución de conflictos, por ejemplo nos referimos a la importancia de contar con figuras cercanas que nos sirvan de modelo y guía de estos procesos.

- Entre las actuaciones comunitarias destacaremos la importancia de considerar el mundo rural enmarcado en un entorno globalizado y en el que se producen dinámicas de cambio. De la gestión responsable de este cambio depende el que mejoremos nuestra calidad de vida y nuestra capacidad para la convivencia.

Las políticas sociales en el medio rural, por ejemplo en el turolense, empiezan a hacerse eco de estas premisas. Desde una perspectiva internacional (los programas Leader, dotados de fondos europeos para el desarrollo económico y social de las regiones), regional (plataforma "Teruel Existe" integra el movimiento asociativo y cívico de la provincia de Teruel y reclama a las institucio- 
nes públicas deudas históricas de dotación institucional e infraestructuras como mejoras en la red ferroviaria, ampliación de la dotación hospitalaria, ampliación de subvenciones públicas, entre otros) y local (el programa local de Aguasvivas invita a inmigrantes argentinos a asentarse en su población, asegurándoles condiciones ventajosas de vivienda y de trabajo durante un determinado tiempo), se intenta dar respuesta a estos retos. En definitiva, el desarrollo rural de manera sostenible aspira a la preservación de su entorno, la continuidad demográfica y la revalorización de su identidad cultural (Balta, 2002).

La equidad y la justicia distributiva constituyen una constante de las definiciones de bienestar en su doble vertiente individual y social. Las políticas públicas pueden mejorar nuestro bienestar, legislando y actuando sobre los factores y los determinantes sociales que generan desigualdad. Su objetivo es facilitar las condiciones sociales bajo las cuales las personas pueden disfrutar de las mismas oportunidades para desarrollar su bienestar (Gil Lacruz, 2007).

La justicia distributiva se puede observar en la igualdad de acceso a los recursos sociales, de las libertades básicas y de las oportunidades para avanzar, así como en la discriminación positiva de los desfavorecidos. La marginación, la exclusión social, la violencia, el maltrato, el fracaso, la discriminación laboral, la dependencia patriarcal y el ma- lestar de los prejuicios culturales constituyen sus opuestos.

La sociedad reclama también proyectos de investigación y de sensibilización social. Programas que nos vacunen contra el machismo, la xenofobia, la violencia o la intolerancia. Educación para la igualdad y la participación responsable. Lo rural ya no puede ser confundido con lo local, lo cerrado o lo autárquico. Ya no se puede establecer una frontera tajante entre las mujeres rurales y urbanas. Por tanto, es el momento de salir al paso de muchos estereotipos falsos que circulan sobre lo rural y sus habitantes, que sobre la base de repetir continuamente que los pueblos desaparecen, que el mundo rural no tiene futuro, que lo que se impone como forma de vida es el modelo urbano, etcétera, nos lo hemos llegado a creer (García Sanz, 1999).

Las mujeres de nuestros pueblos tienen que afrontar los retos de la internacionalización y de un momento histórico en profunda transformación: el desafío de la inmigración, la igualdad de oportunidades, la integración de las nuevas tecnologías, la sociedad de la información, la tercerización de la economía, etcétera. Lo rural ya no es ni el mejor ni el peor de los escenarios. Ni se puede mantener aislado o ensimismado. Aunque siempre la predicción de futuro es incierta, de lo que no cabe ninguna duda es de que su progreso va a depender de la participación activa de sus protagonistas. 


\section{REFERENCIAS}

Altman, M. (2001). Culture, human agency and economic theory: culture as a determinant of material welfare, The Journal of Socioeconomics, 30, 379-391.

Ayuntamiento de Zaragoza (2006). "Plan Municipal de Integración Social y Convivencia Intercultural". Zaragoza: Concejalía de Educación y Acción Social.

Balta, J. (2002). Cultura y desarrollo rural. Ponencia presentada en las Jornadas de Dinamización Sociocultural y Desarrollo Rural. Zaragoza: Universidad de Zaragoza.

Blanco, A. \& Díaz, D. (2005). El bienestar social. En Romay, J.; Salgado, J.; Romaní, M. \& Robla, D. (Comps.). Psicología de las organizaciones, del trabajo y recursos humanos y de la salud (pp. 553560). Madrid: Biblioteca Nueva.

Blanco, A., Rojas, D. \& Corte, L. (2000). La psicología y su compromiso con el bienestar humano. Valencia: Promolibro.

Bourdieu, P. (2002). En defensa de un saber comprometido. Le Monde Diplomatique, 2 de febrero, p. 3.

Campbell, C. (2000). Social capital and health: contextualising health promotion within local community networks. En Campbell, C. \& Jovche- lovitch, S. (Eds.). Health community and development: towards a social psychology of participation, Journal of Community Applied Social Psychology, 10, 255-270.

Díaz, D. \& Blanco, A. (2005). La relación del bienestar subjetivo, bienestar psicológico y bienestar social (coherencia social) con la salud. En Romay, J.; Salgado, J.; Romaní, M. \& Robla, D. (Comps.). Psicología de las organizaciones, del trabajo $y$ recursos humanos y de la salud (pp. 561-573). Madrid: Biblioteca Nueva.

Díaz, E.; Gil, M.; Izquierdo, A.; Jiménez, L.; Lucas, R.; Pérez, I. \& Rezusta, E. (2005). Practicum de psicología social: un modelo comprometido con el entorno. Revista de Humanidades, 11, 257-282.

Frutos, L. M. \& Rubio, P. (2000). Territorio y desarrollo: Los recursos endógenos de la provincia de Teruel. Teruel: Instituto de Estudios Turolenses.

García, J. (2004). Curso de autoempleo para mujeres emprendedoras. Teruel: Universidad de Verano de Teruel.

García, B. (1999). La evolución de la sociedad rural en los últimos 50 años: El papel de la mujer. Ponencia del Congreso Europeo de Mujeres Rurales, Madrid. 
Gil, M. (Ed.). (2007). Psicología social $y$ bienestar. Zaragoza: Prensas Universitarias.

Herrero, J. (2004). La perspectiva ecológica. En Musitu, G.; Herrero, J.; Cantera, L. M. \& M. Montenegro (Eds.). Introducción a la psicología comunitaria (pp. 99-133). Barcelona: Editorial UOC.

Herrero, J.; Cantera, L. M. \& Montenegro, M. (Dirs.). Introducción a la psicología comunitaria (pp. 167-193). Barcelona: UOC.

Izquierdo, A. (2006). Día Internacional de las Mujeres Rurales. Conferencia pronunciada el 15 de octubre en Albarracín: Instituto Aragonés de la Mujer.

Jiménez, T. \& Gil, M. (2007). Psicología comunitaria y plataformas ciudadanas: el reto del bienestar. Revista Iberoamericana de Autogestión y Acción Comunal, 50, 41-58.

Martín, P. \& Agut, S. (2005). La relación entre el individuo y las tecnologías de la información: diferencias de género. Studium Revista de Humanidades, 11, 283-292.
Martínez, E. \& Izquierdo, A. (2007). Bienestar, calidad de vida y desarrollo rural. En Gil, M. (Ed.). Psicología social y bienestar (pp. 285-302). Zaragoza: Prensas Universitarias.

Michalos, A. (1995). Introducción a la teoría de las discrepancias múltiples. Intervención psicosocial, 4, 101-105.

Musitu, G. \& Buelga, S. (2004). Desarrollo y potenciación. En Musitu, G.; Herrero, J.; Cantera, L. \& Montenegro, M. (Eds.). Introducción a la psicología comunitaria. Barcelona: UOC. (2000).

Organización para la Cooperación y Desarrollo (2001): The well being of the nations: the role of human capital and social capital. París: OCDE Publications.

Putnam, R. (1993). Making democracy work. Nueva Jersey: Princeton University Press.

Vásquez, S. (2000) Desarrollo local y territorio. En Pérez, B. \& Carrillo, E. (Coords.). Desarrollo local: manual de uso. Madrid: ESIC. 